\title{
Hypokaliémie par abus de laxatifs
}

Dans le recent numéro de Médecíne et Hygiene (Geneve), lerfévrier 1961), consacré à «Psychosomatique et gastro-entérologie», l’Edito-rial («Гobsédé fécal», p. 65) et plusieurs articles (S. C. Truelove [Oxford], p. 73; R. Dupuy [Paris], p. 74) s'occupent des troubles intestinaux survenant chez les dystoniques neuro-végétatifs, des abus thérapeutiques qu'ils entraînent et notamment des constipations maltraítées. Comme le dit A. McGregor (Brit. Med. J., 7 mai 1960, p. 142) à propos de laxatifs: "The ill-effects of their abuse are far greater than the imagined ill-effects of constipation." On estime que plus de 100 millions d'Américains sont anxieux de leur régularité intestinale, et qu'ils dépensent près de $\$ 150000000$ en laxatifs chaque année. . .

Les consequences des erreurs d'hygiène alimentaire et thérapeu-tique sont généralement bénignes: irritation du gros intestin, apparition d'une endocôlite médicamenteuse, sensible ou franchement douloureuse, qui amène le constipé chez le médecin; Гaffection se corrige facilement une fois le diagnostic correct pose et le traitement adéquat prescrit.

Mais quand on s'obstine à combattre la soi-disant constipation par les laxatifs, il peut en résulter des troubles importants du métabo-lisme. Telle cette «forme spécíale d'hypokaliémie: la dénutrítíonpsycho-genepar abus de laxatífs», à laquelle Ch. Perríer vient de consacrer un important mémoire (Rev. franc, etudes clín. bíol., vol. 5, p. 792, 1960).

Aux 9 observations antérieures parues depuis les deux cas initiaux de A. S. Relman et W. B. Schwartz (New Engl. J. med.; vol. 255, p. 195, 2 août 1956), Perríer ajoute 3 documents de la Clinique thérapeutique de Geneve, particulièrement bien étudiés par la méthode des bilans. En mentionnant encore Гobservation de J. Líchtfield (Gastroentero-logy, vol. 37, p. 483, oct. 1959), on réunit 15 observations caractéri-sées par les signes suivants:

II s'agit le plus souvent de femmes, de tout age (24 à 68 ans), tandis que les 3 hommes étaient toujours des vieillards ( 68 à 78 ans).

Les quantités de laxatifs utilises étaient considerables: 30 dragées à base de séné; 150 à $300 \mathrm{~g}$ de milk of magnesia; $1 \mathrm{~g}$ de cascara 2 à

Editorial

261

3 fois par semaine - et ceci depuis des années (30 et 40 ans parfois), Faction dépurative recherchée étant complétée souvent par des lavements (parfois biquotidiens!) ou par des vomissements provoqués.

Après un certain temps - souvent, hélas, si prolongé qu'on a de la peine à faire la relation entre les habitudes déplorables, mais anciennes, et les phénomènes récents - apparaissent des flatulences, du ballonne-ment, voire un veritable ileus paralytique objective par des «niveaux» à examen radiologique; la fatigue musculaire se traduit par une adynamie profonde, allant parfois jusqu'à la parésie. Ces manifestations entraînent bientôt de $\Gamma$ anorexie qui contraste avec la polydipsie habituelle.

Enfin, ces sujets ont une aptitude particulière à accumuler des ædèmes: la retention d'eau ne se produit que si Гapport aiimentaire en sodium est suffisant. L'ædème présente Гinconvénient 
majeur d'engager le malade à employer un diurétique, qui va le priver encore d'un peu plus de potassium.

En effet, le tableau humoral est tout à fait particulier et caractéris-tique; il comprend:

Un taux de potassium sanguin remarquablement bas: de 1,6 à $2,5 \mathrm{mEq} / 1$ (moyenne $=2,1 \mathrm{mEq} / 1$, au lieu de 4 à $5 \mathrm{mEq} / 1$ normale-ment) car les secretions quotidiennes de $\mathrm{K}$ dans la lumière du tube digestif s'élèvent à $70 \mathrm{mEq}(1,6 \mathrm{~g})$ par 24 heures, quantité correspon-dant à peu près à ce que $\Gamma$ organisme peut trouver dans un regime normal. Mais quand le transit est accéléré au point de produire des selles liquides, celles-ci peuvent contenir jusqu'à $100 \mathrm{mEq}(2,3 \mathrm{~g})$ par jour.

La quantité de $\mathrm{K}$ nécessaire pour rétablir la situation est considerable, variant de 410 à $1090 \mathrm{mEq}$ (en moyenne $681 \mathrm{mEq}=15,7 \mathrm{~g}$ ).

Une alcalose hypochlorhydríque, qui diminue la proportion du calcium ionise, realise un état d'hyperexcitabilité neuro-musculaire; Гeffet paralysant antagoniste de $\Gamma$ hypokaliémie empêche la tétanie de se manifester jusqu'à la phase de correction de la depletion, et cette apparente complication survient quand $\Gamma$ équilibre potassique se rétablit! Le mémoire de Perríer analyse de façon approfondie les mécanismes en jeu et la pathogénie de ce syndrome; mais il insiste sur le fait que Torigine de la maladie est mentale - «horreur de la sensa tion de repletion abdominale» - et le trouble métabolique consécutif. L'explication de cette pathogénie est pour le médecin la meilleure «approche» vis-à-vis d'un malade intelligent et dispense ce dernier de Tintervention du psychiatre. M. D. 